\title{
Disrupted Brain Functional Network Architecture in Chronic Tinnitus Patients
}

\author{
Yu-Chen Chen ${ }^{1 * \dagger}$, Yuan Feng ${ }^{1 \dagger}$, Jin-Jing $X u^{2}$, Cun-Nan Mao ${ }^{1}$, Wenqing Xia ${ }^{3}$, Jun Ren ${ }^{1}$ \\ and Xindao Yin ${ }^{1 *}$ \\ ${ }^{1}$ Department of Radiology, Nanjing First Hospital, Nanjing Medical University, Nanjing, China, ${ }^{2}$ Department \\ of Otolaryngology, Nanjing First Hospital, Nanjing Medical University, Nanjing, China, ${ }^{3}$ Department of Endocrinology, \\ Nanjing First Hospital, Nanjing Medical University, Nanjing, China
}

Purpose: Resting-state functional magnetic resonance imaging (fMRI) studies have demonstrated the disruptions of multiple brain networks in tinnitus patients. Nonetheless, several studies found no differences in network processing between tinnitus patients and healthy controls (HCs). Its neural bases are poorly understood. To identify aberrant brain network architecture involved in chronic tinnitus, we compared the resting-state fMRI (rs-fMRI) patterns of tinnitus patients and HCs.

Materials and Methods: Chronic tinnitus patients $(n=24)$ with normal hearing thresholds and age-, sex-, education- and hearing threshold-matched HCs $(n=22)$ participated in the current study and underwent the rs-fMRI scanning. We used degree

\section{OPEN ACCESS}

Edited by:

Deborah A. Hall,

NIHR Nottingham Hearing Biomedical Research Unit, UK

Reviewed by:

Jeff Edward Davies, De Montfort University, UK

Theo Kypraios,

University of Nottingham, UK

*Correspondence:

Yu-Chen Chen

chenyuchen1989@126.com

Xindao Yin

y.163yy@163.com

${ }^{+}$These authors have contributed equally to this work.

Received: 03 May 2016 Accepted: 28 June 2016 Published: 08 July 2016

Citation:

Chen Y-C, Feng Y, Xu J-J, Mao C-N, Xia W, Ren J and Yin X (2016) Disrupted Brain Functional Network Architecture in Chronic Tinnitus Patients. Front. Aging Neurosci. 8:174. doi: 10.3389/fnagi.2016.00174 centrality (DC) to investigate functional connectivity $(F C)$ strength of the whole-brain network and Granger causality to analyze effective connectivity in order to explore directional aspects involved in tinnitus.

Results: Compared to HCs, we found significantly increased network centrality in bilateral superior frontal gyrus (SFG). Unidirectionally, the left SFG revealed increased effective connectivity to the left middle orbitofrontal cortex (OFC), left posterior lobe of cerebellum (PLC), left postcentral gyrus, and right middle occipital gyrus (MOG) while the right SFG exhibited enhanced effective connectivity to the right supplementary motor area (SMA). In addition, the effective connectivity from the bilateral SFG to the OFC and SMA showed positive correlations with tinnitus distress.

Conclusions: Rs-fMRI provides a new and novel method for identifying aberrant brain network architecture. Chronic tinnitus patients have disrupted FC strength and causal connectivity mostly in non-auditory regions, especially the prefrontal cortex (PFC). The current findings will provide a new perspective for understanding the neuropathophysiological mechanisms in chronic tinnitus.

Keywords: chronic tinnitus, degree centrality, effective connectivity, resting-state fMRI

\section{INTRODUCTION}

Tinnitus is the perception of a sound in the absence of an external sound source. Roughly $12 \%$ of adults experience tinnitus, but the prevalence skyrockets to $50 \%$ in combat personnel (McFadden, 1982; Meikle, 1997). Chronic tinnitus usually leads to problems ranging from mild discomfort such as sleep disturbance to strong anxiety and depression 
(Leske, 1981; Lockwood et al., 2002). Although most tinnitus patients localize tinnitus to one or both ears, the severity of the phantom sound could not be eliminated after sectioning the auditory nerve (Berliner et al., 1992; Jackler and Whinney, 2001). Tinnitus patients show various pathophysiological changes, such as increased spontaneous activity, enhanced neural synchrony, tonotopic map reorganization, abnormal consciousness gating mechanisms and coupling of networks involving auditory and non-auditory structures (Lenarz et al., 1993; Lockwood et al., 1998; Kaltenbach et al., 2005; Adjamian et al., 2009; Henry et al., 2014). Nonetheless, the mechanisms that maintain the disorder remain poorly understood.

Previous neuroimaging studies using electroencephalography (EEG), magnetoencephalography (MEG), positron emission tomography (PET), and functional magnetic resonance imaging (fMRI), have investigated the neuropathophysiological mechanisms implicated in chronic tinnitus (Shulman and Strashun, 1998; Mirz et al., 1999; Adjamian et al., 2009; Schlee et al., 2009; Roberts et al., 2010; Vanneste and De Ridder, 2012). Resting-state fMRI (rs-fMRI) is a promising noninvasive technique that could reflect the brain functional architecture in low frequency fluctuations $(0.01-0.1 \mathrm{~Hz})$ of blood oxygenation level-dependent (BOLD; Biswal et al., 1995; Fox and Raichle, 2007). Using rs-fMRI, multiple brain networks relevant to neural mechanisms of tinnitus have been demonstrated, such as the auditory network (Burton et al., 2012; Kim et al., 2012; Maudoux et al., 2012a,b; Schmidt et al., 2013; Hinkley et al., 2015; Minami et al., 2015; Leaver et al., 2016), dorsal attention network (DAN; Burton et al., 2012; Schmidt et al., 2013), ventral attention network (VAN; Burton et al., 2012), default mode network (DMN; Schmidt et al., 2013; Chen et al., 2014, 2015d; Leaver et al., 2016), and visual network (Burton et al., 2012; Chen et al., 2014). However, these results have been variable due to applied analytic methods. Burton et al. (2012) implied that there is dissociation between activity in auditory cortex and visual, attention and control networks using seed-based functional connectivity (FC) analysis. Through independent component analysis (ICA), Kim et al. (2012) found enhanced FC between the attention network and auditory network, suggesting that this network might contribute to the perception or salience of tinnitus. Schmidt et al. (2013) identified specific alterations in the connectivity of the DMN, DAN, and auditory networks due to tinnitus. Similarly, Maudoux et al. (2012a,b) provided fMRI evidence for a distributed network of auditory and non-auditory cortical and sub-cortical regions associated with chronic tinnitus pathology using ICA approach. Using the algorithms of amplitude of low-frequency fluctuations (ALFF) and regional homogeneity (ReHo), Chen et al. (2014, 2015 c,d) found abnormal spontaneous neural activity within multiple cerebral networks, such as the DMN and the attention network. Nonetheless, other studies have failed to detect any differences in network processing between tinnitus patients and controls (Wineland et al., 2012; Davies et al., 2014). Heterogeneity among tinnitus cohorts may contribute to network state variations. According to these recent theories and observations, we speculated that abnormal brain FC and network might underlie the pathophysiology in chronic tinnitus.

The prefrontal cortex (PFC) exerts early inhibitory modulation of input to primary auditory cortex in humans and has been found to be associated with auditory attention (Lewis et al., 2000; Voisin et al., 2006). The vital role for the PFC in subserving tinnitus mechanism has been postulated (Jastreboff, 1990) and previous neuroimaging studies have confirmed the involvement of PFC for tinnitus (Vanneste et al., 2010, 2012; Vanneste and De Ridder, 2012; De Ridder et al., 2014). Furthermore, latest rs-fMRI studies identified abnormalities in the PFC associated with tinnitus (Burton et al., 2012; Kim et al., 2012; Schmidt et al., 2013; Ueyama et al., 2013; Chen et al., 2014, 2015b,c,d; Leaver et al., 2016). Among them, Ueyama et al. (2013) observed disrupted FC strength in distinct brain regions, especially in the left superior frontal gyrus (SFG), which was negatively correlated with tinnitus loudness. Taken together, the PFC is considered as a key region involved in tinnitus. Investigations exploring the role of PFC in the brain functional network architecture of tinnitus will provide valuable insight into the neural mechanisms underlying the chronic tinnitus.

Seed-based FC and ICA approaches have proved extremely useful in exploring connectivity patterns for specific components of interest. However, few studies have investigated the tinnitusrelated alterations of whole-brain FC pattern or large-scale brain network. Degree centrality (DC) is a voxel-wise datadriven method that can quantify the importance of each node in brain network. This graph theory based network analysis can assess the network centrality without a priori selection of nodes or networks of interest (Zuo et al., 2012). This algorithm has been used to observe the alterations of resting-state functional networks in diverse diseases, such as Alzheimer's disease (AD), autism, and hepatic encephalopathy (Buckner et al., 2009; Di Martino et al., 2013; Chen et al., 2015a). Since the neural mechanisms underlying tinnitus are poorly understood and multiple brain systems are involved, we applied DC to analyze FC within the whole-brain network. To examine the directional connectivity network involved in tinnitus, we further used the granger causality analysis (GCA), which is a statistical method originally used in the field of economics to assess directional influences between simultaneously recorded time series (Granger, 1969; Zhou et al., 2011). GCA has been widely used to reveal the causal effects among brain regions in various neurological or psychiatric disorders, such as AD, schizophrenia, depression and hepatic encephalopathy (Qi et al., 2013; Zhong et al., 2014; Guo et al., $2015 a, b)$. Thus, to unravel the details of the brain functional network architecture in tinnitus, we sought to evaluate the brain regions that show aberrant FC across the entire brain networks in tinnitus patients using DC and then use GCA to analyze effective connectivity to understand the directional aspect of these alterations. We hypothesized that the intrinsic dysconnectivity pattern of the PFC might play a crucial role in the brain functional network architecture of tinnitus patients. 


\section{MATERIALS AND METHODS}

\section{Subjects}

All the subjects provided written informed consent before their participation in the study protocol, which was approved by the Research Ethics Committee of the Nanjing Medical University (Reference No. 2016067).

According to both the inclusion and exclusion criteria of this study, a final sample of 47 subjects including 25 chronic tinnitus patients and 22 healthy controls (HCs) were recruited through community health screening and newspaper advertisements. The tinnitus patients and healthy subjects were group-matched in terms of age, sex, and education. One tinnitus patient was subsequently excluded because the limits for head motion were exceeded during MR scanning. Ten patients reported a predominantly left-sided tinnitus, six a predominantly rightsided tinnitus and eight patients described their tinnitus as bilateral or originating within the head. All subjects were righthanded and completed at least 8 years of education. The severity of tinnitus and related distress were assessed by the Iowa version of the Tinnitus Handicap Questionnaires (THQ; Kuk et al., 1990). Hearing thresholds were determined by puretone audiometry (PTA). All of the participants had normal hearing (defined as thresholds $<25 \mathrm{~dB} \mathrm{HL}$ ) at the frequencies of $0.25 \mathrm{kHz}$, $0.5 \mathrm{kHz}, 1 \mathrm{kHz}, 2 \mathrm{kHz}, 4 \mathrm{kHz}$, and $8 \mathrm{kHz}$. There were no significant differences in auditory thresholds between tinnitus and control groups. In addition, none of the participants had symptoms of depression and anxiety according to the Self-Rating Depression Scale (SDS) and Self-Rating Anxiety Scale (SAS; overall scores <50, respectively; Zung, 1971, 1986). According to previous study (Khalfa et al., 2002), we used the Hyperacusis Questionnaire to exclude the participants with hyperacusis in the current study. Participants were also excluded from the study if they suffered from pulsatile tinnitus or Meniere's diseases, or if they had a past history of severe smoking, stroke, alcoholism, brain injury, Parkinson's disease, AD, epilepsy, major depression, neurological or psychiatric disorders that could affect cognitive function, major medical illness (e.g., anemia, thyroid dysfunction and cancer), MRI contraindications (e.g., cochlear implants, pacemakers, cerebral aneurysm clips, prosthetic valves, a history of intraocular metal fragments, and claustrophobia), or severe visual loss. The characteristics of the chronic tinnitus patients and healthy subjects are summarized in Table $\mathbf{1 .}$

TABLE 1 | Characteristics of the tinnitus patients and healthy controls (HCs).

\begin{tabular}{lccc}
\hline & $\begin{array}{c}\text { Tinnitus patients } \\
(\boldsymbol{n}=\mathbf{2 4})\end{array}$ & $\begin{array}{c}\text { HCs } \\
(\boldsymbol{n}=\mathbf{2 2})\end{array}$ & $\boldsymbol{p}$ value \\
\hline Age (year) & $50.8 \pm 12.4(26-67)$ & $44.7 \pm 15.4(26-70)$ & 0.144 \\
Gender (male: female) & $9: 15$ & $9: 13$ & 0.813 \\
Education levels (years) & $12.3 \pm 3.1(8-18)$ & $13.4 \pm 3.8(8-22)$ & 0.313 \\
Tinnitus duration (months) & $46.5 \pm 39.1(6-120)$ & - & - \\
THQ score & $49.5 \pm 15.5$ & - & - \\
Hearing thresholds (left) & $13.0 \pm 2.7$ & $13.6 \pm 2.2$ & 0.414 \\
Hearing thresholds (right) & $14.6 \pm 3.6$ & $13.9 \pm 3.4$ & 0.470 \\
\hline
\end{tabular}

Data are represented as Mean $\pm S D$ (range of min-max).

\section{MRI Acquisition}

MRI data were acquired at our hospital using a $3.0 \mathrm{~T}$ MRI scanner (Ingenia, Philips Medical Systems, Netherlands). Head motion and scanner noise were reduced using foam padding and earplugs. The earplugs (Hearos Ultimate Softness Series, USA) were used to attenuate scanner noise by approximately $32 \mathrm{~dB}$. Subjects were instructed to lie quietly with their eyes closed without falling asleep, not think of anything in particular, and avoid any head motion during the scan. Functional images were obtained axially using a gradient echo-planar imaging (EPI) sequence as follows: repetition time $(\mathrm{TR})=2000 \mathrm{~ms}$; echo time $(\mathrm{TE})=30 \mathrm{~ms}$; slices $=36$; thickness $=4 \mathrm{~mm}$; gap $=0 \mathrm{~mm}$; field of view $(\mathrm{FOV})=240 \mathrm{~mm} \times 240 \mathrm{~mm}$; acquisition matrix $=64 \times 64$; and flip angle $(\mathrm{FA})=90^{\circ}$. The fMRI sequence took $8 \mathrm{~min}$ and 8 s. Structural images were acquired with a three-dimensional turbo fast echo (3D-TFE) T1WI sequence with high resolution as follows: $\mathrm{TR} / \mathrm{TE}=8.1 / 3.7 \mathrm{~ms}$; slices $=170$; thickness $=1 \mathrm{~mm}$; gap $=0 \mathrm{~mm} ; \mathrm{FA}=8^{\circ} ;$ acquisition matrix $=256 \times 256$; FOV $=$ $256 \mathrm{~mm} \times 256 \mathrm{~mm}$. The structural sequence took $5 \mathrm{~min}$ and $29 \mathrm{~s}$.

\section{Functional Data Preprocessing}

Functional data analyses were conducted using Data Processing Assistant for Resting-State fMRI (DPARSF) programs (ChaoGan and Yu-Feng, 2010) based on statistical parametric mapping $\left(\mathrm{SPM}^{1}\right)$ and rs-fMRI data analyses toolkits $\left(\mathrm{REST}^{2}\right)$. A total of 240 volumes were scanned, and the first 10 volumes were discarded to allow for signal equilibrium of the initial magnetic resonance signals and adaptation of the subjects to scanner. The remaining 230 consecutive volumes were used for data analysis. Afterwards, the following procedures were carried out as follows: slice-timing adjustment, realignment for head-motion correction, spatial normalization to the Montreal Neurological Institute $(\mathrm{MNI})$ template (resampling voxel size $=3 \times 3 \times$ $3 \mathrm{~mm}^{3}$ ) and smoothing with an isotropic Gaussian kernel (full width at half maximum (FWHM) $=6 \mathrm{~mm}$ ), detrending and filtering (0.01-0.08 Hz). Any subjects with a head motion $>2.0 \mathrm{~mm}$ translation or a $2.0^{\circ}$ rotation in any direction were excluded.

\section{Degree Centrality Analysis}

We restricted our voxel-wise centrality analyses to a predefined gray matter (GM) mask that included tissue with GM probabilities greater than $20 \%$ as previously described (Zuo et al., 2012). Within the mask, individual network centrality maps were generated in a voxel-wise fashion. First, the preprocessed functional runs were subjected to voxel-based whole-brain correlation analysis. The time course of each voxel from each participant was correlated with the time course of every other voxel, which resulted in a correlation matrix. An undirected adjacency matrix was then obtained by thresholding each correlation at $r>0.25$ (Buckner et al., 2009; Zuo et al., 2012; Yan et al., 2013). As previously reported, the negative correlations were not included in DC calculation, given their ambiguous interpretation and detrimental effects

\footnotetext{
${ }^{1}$ http://www.fil.ion.ucl.ac.uk/spm

${ }^{2}$ http://www.restfmri.net
} 
on test-retest reliability (Buckner et al., 2008; Vincent et al., 2008; Murphy et al., 2009). A high threshold was chosen to eliminate counting voxels that had low temporal correlation attributable to signal noise. Different threshold selections did not qualitatively change the results for cortex (Buckner et al., 2009). Then, the DC was computed as the number of significant correlations (binarized) or as the sum of the weights of the significant connections (weighted) for each voxel (Buckner et al., 2009; Zuo et al., 2012). This measure of connectivity (degree, $D$ ) for each voxel $(i)$ with all other voxels $(j)$ is given by the following: $D_{i}=\sum d_{i j}$ where, $j=1 \ldots N$, $i \neq j$. The map of the connectivity was then standardized by converting to $z$ scores so that maps across participants could be averaged and compared. The $z$ score transformation is given by:

$$
Z_{i}=\frac{D_{i}-\bar{D}}{\sigma_{D}} i=1 \ldots N
$$

The $\bar{D}$ is the mean degree across all the voxels in the whole-brain map and $\sigma_{D}$ is the standard deviation of the map. DC has been shown to represent the most local and directly quantifiable centrality measure and has been widely used to examine node characteristics of intrinsic network connectivity (Zuo et al., 2012). The DC maps were transferred to $z$-values for group comparisons. Within brain network, the DC value of a node indicates its connectivity strength to all the other nodes and reflects its importance in functional integration.

We first estimated spatial distribution of mean DC in the tinnitus group and healthy group, respectively. The individual $z$ values were entered into the SPM8 software for a random effect one-sample $t$-test in a voxel-wise way to show the average DC maps within each group. The significant threshold was set at $p<0.01$, with multiple comparisons correction using the AFNI AlphaSim program ${ }^{3}$ determined by Monte Carlo simulation (AlphaSim program with following parameters: single voxel $p$ value of 0.01 , a minimum cluster size of 40 voxels, 5000 simulations, cluster connection radius $r=5 \mathrm{~mm}$, FWHM $=$ $6 \mathrm{~mm}$ ). This correction was confined within the aforementioned GM mask.

To find the disrupted brain hub regions, two-tailed twosample $t$-test were then conducted to investigate the differences in the DC maps between tinnitus patients and HCs. Betweengroup comparisons of the DC maps were performed in the SPM8 software using general linear model (GLM) analysis, with age, sex and education included as nuisance covariates. A correction for multiple comparisons was performed by a Monte Carlo simulation using the AlphaSim program, resulting in a corrected threshold of $p<0.01$ and minimum cluster size of 40 voxels (parameters were single voxel $p$ value of $0.01,5000$ simulations, cluster connection radius $r=5 \mathrm{~mm}, \mathrm{FWHM}=6 \mathrm{~mm}$ ). For between-group analysis, a mask was created by combining the significant clusters in both groups, which were obtained from one-sample $t$-test results.

\footnotetext{
${ }^{3}$ https://afni.nimh.nih.gov/pub/dist/doc/manual/AlphaSim.pdf
}

\section{Effective Connectivity Analysis}

Using DC approach, we were able to show that the bilateral SFG is a region of special functional importance in tinnitus patients revealing increased FC. To further investigate the influence of directionality, we applied GCA to evaluate changes in effective connectivity. Based on the results of the DC analysis we selected the seed regions, which showed significant differences between tinnitus patients and HCs (left and right SFG: MNI coordinates $(x, y, z) \pm 18,42,27)$. Effective connectivity was analyzed using REST-GCA in the REST toolbox (Zang et al., 2012). In this study, two separate time series of the left and right SFG were defined as the seed time series $x$, and the time series $y$ denotes the time series of all voxels in the brain. The linear direct influence of $x$ on $y\left(\mathrm{~F}_{x \rightarrow y}\right)$, and the linear direct influence of $y$ on $x$ $\left(\mathrm{F}_{y \rightarrow x}\right)$ were calculated voxel by voxel across the brain. Thus, two Granger causality maps were generated based on the influence measures for each subjects. The residual-based $F$ was normalized $\left(\mathrm{F}^{\prime}\right)$ and standardized to $\mathrm{Z}$ score for each voxel $\left(\mathrm{Z}_{x \rightarrow y}\right.$ and $\mathrm{Z}_{y \rightarrow x}$, subtracting the global mean $\mathrm{F}^{\prime}$ values, divided by standard deviation).

For the group analysis on the effective connectivity, mean values of $Z_{x \rightarrow y}$ and $Z_{y \rightarrow x}$ maps were computed for each group. All eight Granger causality maps were acquired, with four for each direction and four for each group (the left SFG with $Z_{x \rightarrow y}$ and $Z_{y \rightarrow x}$ and the right SFG with $Z_{x \rightarrow y}$ and $Z_{y \rightarrow x}$ for both tinnitus patient and HCs). Then these Granger causality maps were entered into SPM8 software for group comparison. A random effect two-sample $t$-test in a voxel-wise manner was performed to determine the differences of effective connectivity of the SFG between tinnitus patient and HCs, with age, sex and education including as nuisance covariates. A correction for multiple comparisons was also conducted by a Monte Carlo simulation using the AlphaSim program, with a corrected threshold of $p<0.01$ and minimum cluster size of 40 voxels.

\section{Correlation Analysis}

To investigate the relationship between clinical characteristic of tinnitus patients and DC and effective connectivity measures, these regions showing significant differences in DC or effective connectivity between groups were extracted. Mean $z$ values within these clusters were correlated against each tinnitus characteristic using the Pearson's correlation analysis by SPSS software (version 18.0; SPSS, Chicago, IL, USA). $P<0.05$ was considered statistically significant, corrected for age, sex and education. Bonferroni correction for multiple comparisons was applied in the correlation analysis.

\section{RESULTS}

\section{Degree Centrality Analysis}

In both HC (Figure 1A) and tinnitus patients (Figure 1B), the spatial distribution of the weighted DC was highly localized in the occipital lobe, inferior parietal lobe (IPL), PFC, cingulate cortex, insula and thalamus. After two-sample $t$-test analysis, significantly increased DC within the bilateral SFG was found 


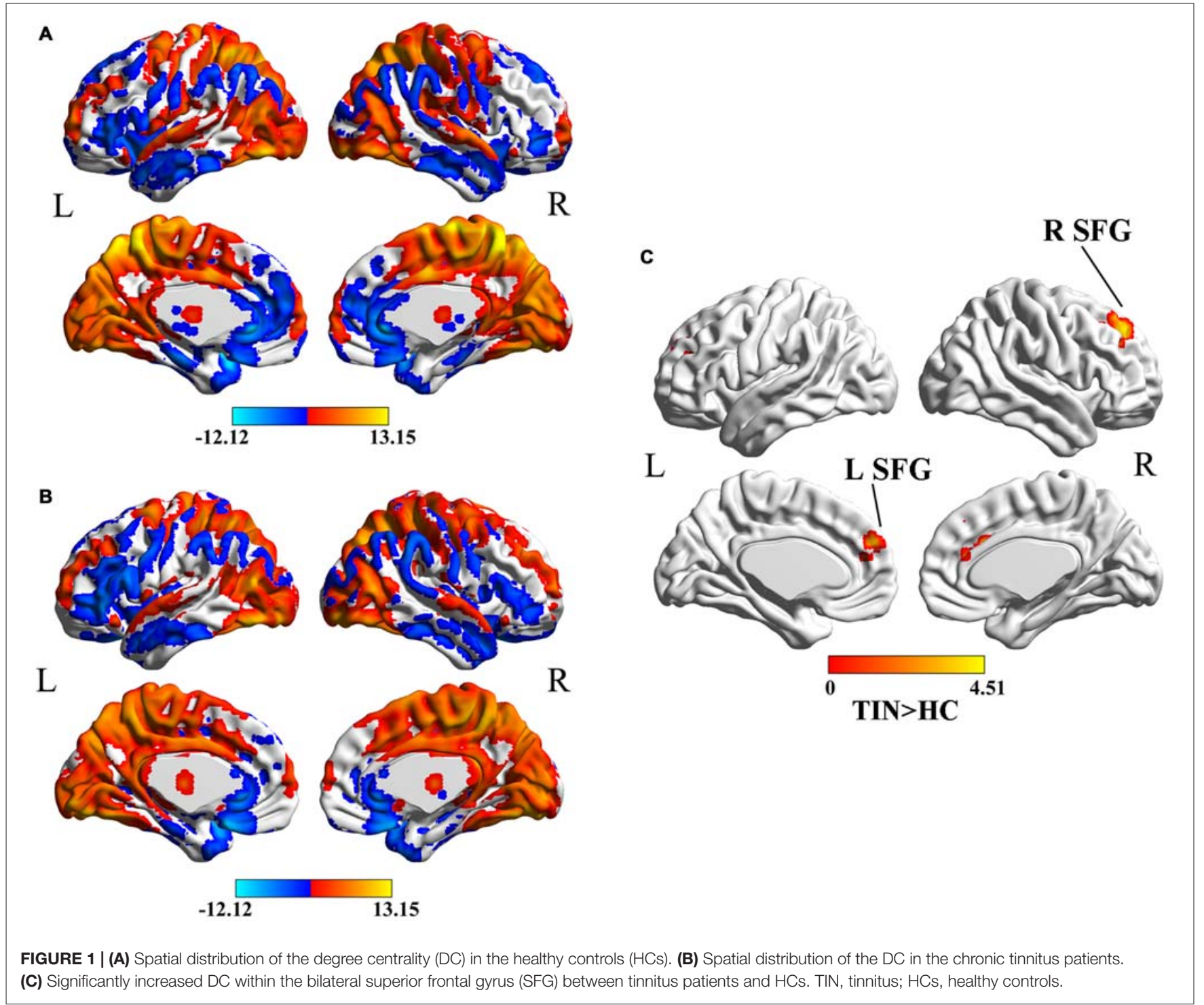

in tinnitus patients compared to HCs $(p<0.01$, AlphaSim corrected; Figure 1C).

\section{Effective Connectivity Analysis}

Compared to HCs, patients with chronic tinnitus demonstrated significantly increased effective connectivity from the left SFG to the left middle orbitofrontal cortex (OFC), left posterior lobe of cerebellum (PLC), left postcentral gyrus (PoCG), and right middle occipital gyrus (MOG). Moreover, the right SFG exhibited enhanced effective connectivity to the right supplementary motor area (SMA; $p<0.01$, AlphaSim corrected; Figure 2 and Table 2). However, we did not find any abnormal feedback effect to the bilateral SFG in the tinnitus patients.

\section{Correlation Results}

We found no significant correlations between the DC of the bilateral SFG and the tinnitus characteristics. However, the
THQ scores positively correlated with the increased effective connectivity from the left SFG to left OFC ( $r=0.504, p=0.020)$, and from the right SFG to right SMA $(r=0.526, p=0.014$; Figure 3 ). The other regions with enhanced effective connectivity revealed no significant correlations with tinnitus duration or THQ scores.

\section{DISCUSSION}

This is the first study to use both DC and GCA approaches to explore intrinsic functional network architecture related to tinnitus. Using DC analysis, we found significantly increased network centrality within the bilateral SFG regions in chronic tinnitus patients. Using GCA algorithm, we employed the bilateral SFG as seeds to examine their causal effect with the whole brain. Unidirectionally, bilateral SFG showed increased effective connectivity to several non-auditory regions including the prefrontal, motor, visual cortex and cerebellum. Of note, 

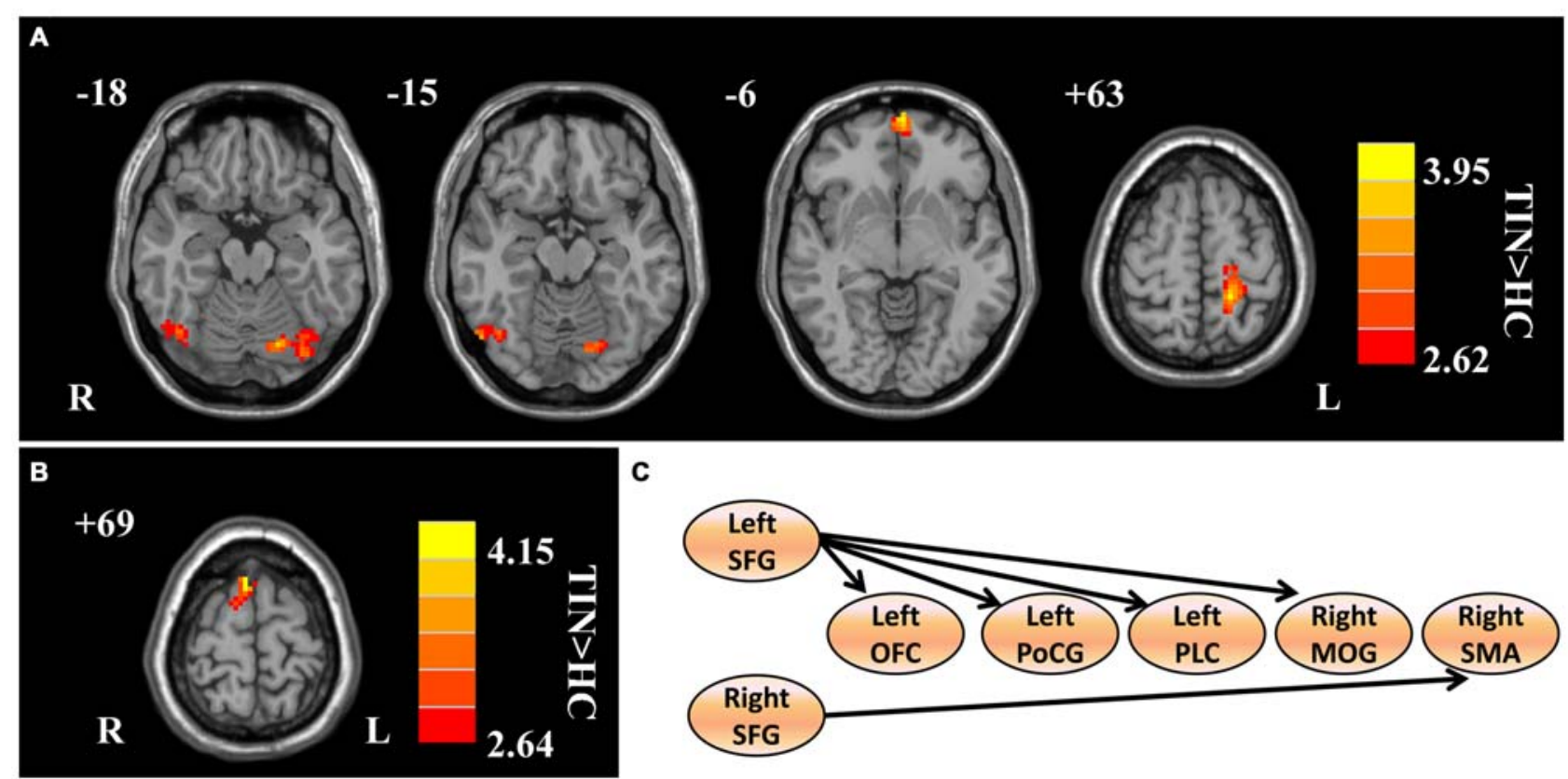

C

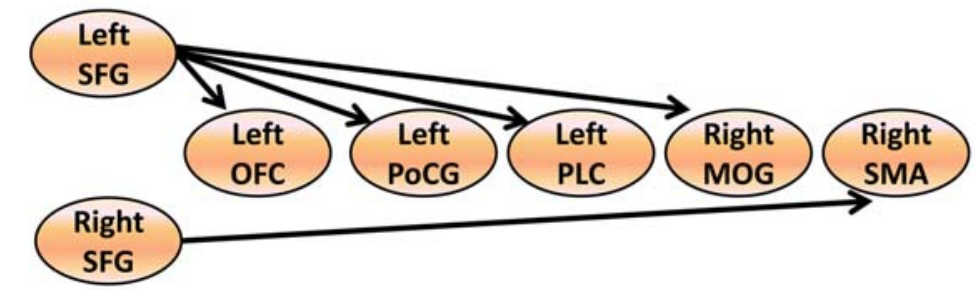

FIGURE 2 | Aberrant effective connectivity from the bilateral SFG in tinnitus patients. (A) Increased effective connectivity from the left SFG to the left orbitofrontal cortex (OFC), left PoCG, left posterior lobe of cerebellum (PLC), and right middle occipital gyrus (MOG; $p<0.01$, AlphaSim corrected). (B) Increased effective connectivity from the right SFG to the right supplementary motor area (SMA; $p<0.01$, AlphaSim corrected). (C) Schematic overview of changes in effective connectivity from the bilateral SFG. SFG, superior frontal gyrus; OFC, orbitofrontal cortex; PoCG, precentral gyrus; PLC, posterior lobe of cerebellum; MOG, middle occipital gyrus; SMA, supplementary motor area; TIN, tinnitus; HCs, healthy controls.

the abnormal connectivity to the OFC and the SMA revealed significant correlations with the tinnitus distress.

\section{Increased Network Centrality in the SFG}

Certain regions show strong connections with other regions within large-scale cortical networks that constitute an emerging feature of brain architecture (Sporns et al., 2007; Buckner et al., 2009; Zuo et al., 2012). Our findings show that the SFG is the main cortical hub in the brain network architecture affected by tinnitus, which is in line with the

TABLE 2 | Regions showing significant differences in effective connectivity between tinnitus patients and HCs.

\begin{tabular}{|c|c|c|c|c|}
\hline Brain regions & BA & $\begin{array}{c}\text { Peak MNI coordinates } \\
\qquad x, y, z(\mathrm{~mm})\end{array}$ & $\begin{array}{c}\text { Peak } \\
T \text { value }\end{array}$ & Voxels \\
\hline
\end{tabular}

Increased effective connectivity from left SFG

$\begin{array}{llllll}\text { Left OFC } & 11 & -3, & 66,-6 & 4.1302 & 158\end{array}$

Left POCG

Left PLC

Right MOG

$-\quad-18,-75,-18$

$1954,-69,-15$

4.0470

132

$3.6185 \quad 46$

Increased effective connectivity from right SFG

$\begin{array}{llllll}\text { Right SMA } & 6 & 6, & 15, & 69 & 4.4562\end{array}$

A corrected threshold of $p<0.01$ was determined by Monte Carlo simulation. BA, Brodmann's area; MNI, Montreal Neurological Institute; SFG, superior frontal gyrus; OFC, orbitofrontal cortex; PoCG, precentral gyrus; PLC, posterior lobe of cerebellum; MOG, middle occipital gyrus; SMA, supplementary motor area. hypothesis that the dysconnectivity pattern of the PFC involved in tinnitus perception. The PFC has been regarded as a critical region by Jastreboff (1990), who suggested that the PFC integrates sensory and emotional aspects of tinnitus. Several neurophysiological models based on neuroimaging have been raised involved in tinnitus subsequently (De Ridder et al., 2014). Rauschecker et al. (2010) developed a model to demonstrate structural and functional differences in ventromedial prefrontal cortex (vmPFC) that were associated with tinnitus subjective loudness, indicating that PFC may contribute to certain perceptual features of tinnitus (Leaver et al., 2011). The current integrative model attempts to unify the different brain areas and networks associated with tinnitus in one model and propose the hypothetical neural core of conscious phantom sound perception (De Ridder et al., 2011, 2014; Vanneste and De Ridder, 2012), which is more extensive than the initially proposed auditory system (Jastreboff and Hazell, 2008).

Our current study suggested that the PFC, specifically the SFG, might be a major integrative hub of the model that should be involved to perceive tinnitus. However, we did not observe any abnormal neural activity in auditory system. The most parsimonious explanation for this is the absence of any hearing loss out to $8 \mathrm{kHz}$ and the absence of hyperacusis in our tinnitus patients. We speculated that aberrant neural activity of the PFC may exist prior to the disruption of the auditory system in tinnitus patients with normal hearing. In addition, since our tinnitus patients showed no obvious symptoms of depression, 
anxiety or cognitive decline, we did not detect any abnormalities in the limbic system. Based on previous fMRI studies, the SFG is a significant part of auditory connection cortex that can receive and integrate all kinds of information from different parts of the brain from inside and outside the body. Besides, it can also timely organize efferent impulses to ensure the coordination of the central nervous system (CNS) as a whole (Mathiak et al., 2007; Melloni et al., 2007). A possible interpretation for our results was that the increased DC in bilateral SFG might be due to feedback inhibition of the over activity in auditory network.

Previous neuroimaging studies have pointed out that the abnormalities of the SFG could act as a direct mechanism of tinnitus chronification (Giraud et al., 1999; Mirz et al., 1999; Haller et al., 2010). Wunderlich et al. (2010) detected the SFG activation after acoustic stimulation in a pitch discrimination task, suggesting the perception of auditory inputs in a more emotional context due to tinnitus. Chen et al. (2014) found increased ALFF values in the right SFG in chronic tinnitus patients, which was linked with tinnitus duration and distress. The SFG was also proved to be influenced by chronic tinnitus in recent rs-fMRI studies (Maudoux et al., 2012a; Ueyama et al., 2013; Chen et al., 2015c; Zhang et al., 2015). In line with these valuable findings, our results have implications for understanding the specific role of SFG abnormalities in chronic tinnitus, suggesting the PFC may provide valuable insight into the neural mechanisms underlying tinnitus.

\section{Increased Effective Connectivity from the SFG}

Using a GCA method, the present study exhibited that the information flow is unidirectionally affected (seed to whole brain) within non-auditory areas due to tinnitus. We found increased influence from the SFG to the frontal cortex (OFC and SMA), cerebellum (PLC), visual cortex (MOG) and somatosensory cortex (PoCG). Previous neuroimaging studies have identified abnormalities in the frontal cortex which could account for tinnitus. Firstly, it has been revealed that the OFC is critical for emotional processing of sounds (Damasio et al., 1996; Blood et al., 1999). The alterations of the OFC have been found in the functional coupling of long-range cortical networks between tinnitus patients and HCs by using EEG or MEG (Schlee et al., 2009; Vanneste et al., 2012; Song et al., 2014). Moreover, recent rs-fMRI studies have also demonstrated the abnormal FC of the OFC in tinnitus patients (Maudoux et al., 2012a; Chen et al., 2015d; Zhang et al., 2015). The OFC is also regarded as part of the reward system, which might integrate the aversive information of the perceived tinnitus (Rolls, 2004; Kringelbach, 2005). The heightened effective connectivity from the SFG to the OFC might be interpreted as a dysfunctional inhibitory response directing attention away from phantom sound perception. Our finding of positive correlation between the effective connection from the SFG to the OFC and tinnitus distress also emphasized the pivotal role of the OFC in tinnitus. Furthermore, we also found positive correlation between the THQ score and increased influence from the SFG to the SMA, which is regarded as a part of the primate cerebral cortex that contributes to the control of movement (Roland et al., 1980). By using quantitative EEG (qEEG), Vanneste et al. (2011) observed aberrant neuronal activity in the SMA in unilateral or bilateral tinnitus (Vanneste and De Ridder, 2012). It was hypothesized that synchronized theta activity in the SMA might be accountable for part of the conscious perception of the phantom sound (Vanneste and De Ridder, 2012). Nevertheless, further research is needed to clarify the role of the SMA in tinnitus.

Although the cerebellum is primarily involved in motor actions and control, some cerebellar regions such as the paraflocculus and vermis receive inputs from auditory centers (Petacchi et al., 2005). Auditory sensory processing in the cerebellum has been reported. Osaki et al. (2005) revealed that the right cerebellum was involved in tinnitus and showed a decreased regional blood flow during residual inhibition. Using rs-fMRI, chronic tinnitus patients showed increased FC in the cerebellar hemisphere that was linked with tinnitus distress (Maudoux et al., 2012a; Ueyama et al., 2013), confirming the involvement of cerebellum in auditory system. Nevertheless, the association of the cerebellum with tinnitus has not been substantially elucidated. Moreover, increased effective connectivity to MOG and PoCG raised the question of whether tinnitus might be linked to phantom visual or somatosensory perceptions. Such changes seem reasonable given the multisensory interactions known to exist between auditory, visual and somatosensory regions. Possible neural correlates of visual or somatosensory modulation of tinnitus were assessed (Murray et al., 2005). One interpretation of these results is that as patients attend to their phantom auditory sensation they contemporaneously activate visual or somatosensory areas. This interpretation is consistent with previous rs-fMRI studies showing abnormal neural activity in visual network (Burton et al., 2012; Maudoux et al., 2012a; Chen et al., 2014, 2015b,c,d) or somatosensory network (Maudoux et al., 2012a; Ueyama et al., 2013) in tinnitus. Therefore, tinnitus can be regarded as the consequence of multiple brain subnetworks involved in the different aspects of tinnitus, both acoustic and affective.

\section{Tinnitus Lateralization}

Interestingly, results of effective connectivity analysis were lateralized to the left hemisphere region in tinnitus patients. Asymmetry for the tinnitus patients has been reported both structurally and functionally (Mühlau et al., 2006; Smits et al., 2007; Schecklmann et al., 2013; Chen et al., 2014). Previous PET studies have demonstrated an overactivation of the left auditory cortex independent of tinnitus laterality and anatomical hemispheric differences (Arnold et al., 1996; Langguth et al., 2006; Schecklmann et al., 2013). This lateralization may be explained as an increase in activation on the side of the perceived tinnitus, or a decrease in activation on the side contralateral to the side of perceived tinnitus. The interpretation would indicate an increased spontaneous neural activity of the affected brain area in tinnitus patients (Smits et al., 2007). However, several studies also confirmed the right- 

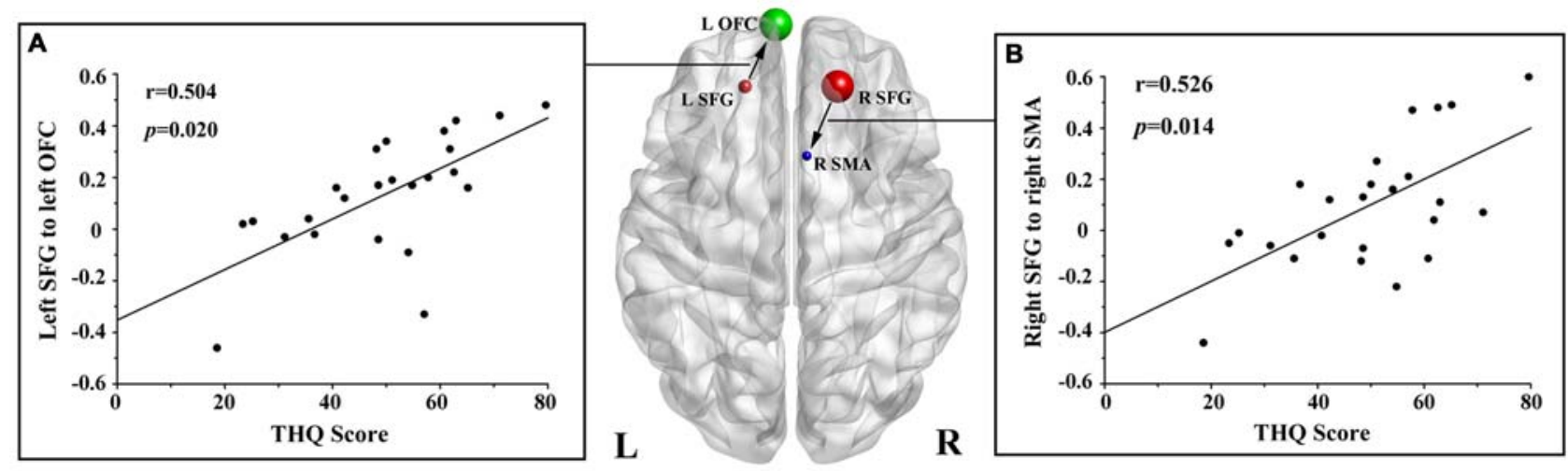

FIGURE 3 | Correlations between the increased effective connectivity of the bilateral SFG and the tinnitus handicap questionnaires (THQ) scores. (A) The THQ scores positively correlated with the increased effective connectivity from the left SFG to left OFC $(r=0.504, p=0.020)$. (B) The THQ scores positively correlated with the increased effective connectivity from the right SFG to right SMA ( $r=0.526, p=0.014)$. L, left; R, right; THQ, tinnitus handicap questionnaires; SFG, superior frontal gyrus; OFC, orbitofrontal cortex; SMA, supplementary motor area.

lateralization in tinnitus (Chen et al., 2014; Geven et al., 2014). The inconsistencies between studies may be due to the different neuroimaging methods used to investigate tinnitus or heterogeneity of the tinnitus patients. Therefore, further studies are required to determine if the observed left hemispheric dominance is related specifically to tinnitus or some other factors.

\section{Limitations}

Study limitations must be acknowledged. First, we admit that it is difficult to make direct causal inferences regarding the relationships between the brain functional network architecture and tinnitus characteristics in tinnitus patients, considering the cross-sectional nature of our experimental design and limited sample size. Further longitudinal studies involving a greater number of subjects are required. The very nature of BOLDfMRI makes it difficult to measure resting-state network activity given that amplitude is not measured only correlations of BOLD signals (Logothetis et al., 2001). The relationship between the measured BOLD-fMRI signal and the underlying neural activity is still largely unknown. Further studies combining fMRI with EEG may help understand the exact relationship. Second, common criticisms regarding GCA algorithm for rs-fMRI data have been raised. For example, the fallacious effects might occur because of the systematic differences across brain regions in hemodynamic delays. GCA has been confounded by the presence of vascular anatomy (Webb et al., 2013). Additionally, changes in directionality could be caused by the differences in hemodynamic coupling in different regions (Smith et al., 2011). Furthermore, we attempted to exclude subjects with hyperacusis from our study because subjects with hyperacusis exhibited robust activation in selected brain regions, such as the primary auditory cortex, thalamus, and auditory midbrain (Gu et al., 2010). However, it would be useful to include subjects with hyperacusis in future studies so as to examine if intrinsic functional network architecture is disrupted in a similar manner to that was observed in our tinnitus patients without hyperacusis.
Finally, although we attempt to reduce the MR scanner noise with earplugs, the subjects cannot be completely prevented from hearing some noise that probably alters the resting-state brain networks to varying degree (Logothetis et al., 2009). This confounding factor should be taken into consideration for all rsfMRI studies related to the auditory systems.

\section{CONCLUSION}

In this study, we show that disrupted functional network architecture within non-auditory regions revealed by DC and GCA are present in chronic tinnitus patients with normal hearing, without hyperacusis. Chronic tinnitus patients exhibited enhanced FC strength in bilateral SFG regions, which showed unidirectionally increased causal connectivity to frontal, motor, visual, somatosensory cortex and cerebellum. Furthermore, increased effective connectivity from bilateral SFG to OFC and SMA were positively correlated with tinnitus distress. Disturbance in brain functional network architecture will contribute to a better understanding of the neuropathophysiological mechanisms in tinnitus perception.

\section{AUTHOR CONTRIBUTIONS}

Y-CC and YF designed the experiment, collected the data, performed the analysis and wrote the manuscript. J-JX and C-NM collected the data. WX, JR, and XY contributed to the discussion and manuscript revision.

\section{ACKNOWLEDGMENTS}

The authors thank Prof. Richard Salvi, Center for Hearing and Deafness, University at Buffalo, Buffalo, NY, USA, for his contributions to the design of the study. This work was supported by the Collaborative Innovation Center of Suzhou Nano Science and Technology. 


\section{REFERENCES}

Adjamian, P., Sereda, M., and Hall, D. A. (2009). The mechanisms of tinnitus: perspectives from human functional neuroimaging. Hear. Res. 253, 15-31. doi: 10.1016/j.heares.2009.04.001

Arnold, W., Bartenstein, P., Oestreicher, E., Römer, W., and Schwaiger, M. (1996). Focal metabolic activation in the predominant left auditory cortex in patients suffering from tinnitus: a PET study with $[18 \mathrm{~F}]$ deoxyglucose. ORL J. Otorhinolaryngol. Relat. Spec. 58, 195-199. doi: 10.1159/000276835

Berliner, K. I., Shelton, C., Hitselberger, W. E., and Luxford, W. M. (1992). Acoustic tumors: effect of surgical removal on tinnitus. Otol. Neurotol. 13, 13-17. doi: 10.1097/00129492-199201000-00005

Biswal, B., Zerrin Yetkin, F., Haughton, V. M., and Hyde, J. S. (1995). Functional connectivity in the motor cortex of resting human brain using echo-planar MRI. Magn. Reson. Med. 34, 537-541. doi: 10.1002/mrm. 1910340409

Blood, A. J., Zatorre, R. J., Bermudez, P., and Evans, A. C. (1999). Emotional responses to pleasant and unpleasant music correlate with activity in paralimbic brain regions. Nat. Neurosci. 2, 382-387.

Buckner, R. L., Andrews-Hanna, J. R., and Schacter, D. L. (2008). The brain's default network. Ann. N Y Acad. Sci. 1124, 1-38. doi: 10.1196/annals. 1440.011

Buckner, R. L., Sepulcre, J., Talukdar, T., Krienen, F. M., Liu, H., Hedden, T., et al. (2009). Cortical hubs revealed by intrinsic functional connectivity: mapping, assessment of stability and relation to Alzheimer's disease. J. Neurosci. 29, 1860-1873. doi: 10.1523/jneurosci.5062-08.2009

Burton, H., Wineland, A., Bhattacharya, M., Nicklaus, J., Garcia, K. S., and Piccirillo, J. F. (2012). Altered networks in bothersome tinnitus: a functional connectivity study. BMC Neurosci. 13:3. doi: 10.1186/1471-2202-13-3

Chao-Gan, Y., and Yu-Feng, Z. (2010). DPARSF: a MATLAB toolbox for "pipeline" data analysis of resting-state fMRI. Front. Syst. Neurosci. 4:13. doi: 10.3389/fnsys.2010.00013

Chen, H.-J., Jiang, L.-F., Sun, T., Liu, J., Chen, Q.-F., and Shi, H.-B. (2015a). Resting-state functional connectivity abnormalities correlate with psychometric hepatic encephalopathy score in cirrhosis. Eur. J. Radiol. 84, 2287-2295. doi: 10.1016/j.ejrad.2015.08.005

Chen, Y.-C., Xia, W., Feng, Y., Li, X., Zhang, J., Feng, X., et al. (2015b). Altered interhemispheric functional coordination in chronic tinnitus patients. Biomed. Res. Int. 2015:345647. doi: 10.1155/2015/345647

Chen, Y.-C., Xia, W., Luo, B., Muthaiah, V. P., Xiong, Z., Zhang, J., et al. (2015c). Frequency-specific alternations in the amplitude of low-frequency fluctuations in chronic tinnitus. Front. Neural Circuits 9:67. doi: 10.3389/fncir.2015.00067

Chen, Y.-C., Zhang, J., Li, X.-W., Xia, W., Feng, X., Qian, C., et al. (2015d). Altered intra-and interregional synchronization in resting-state cerebral networks associated with chronic tinnitus. Neural Plast. 2015:475382. doi: 10. 1155/2015/475382

Chen, Y.-C., Zhang, J., Li, X.-W., Xia, W., Feng, X., Gao, B., et al. (2014). Aberrant spontaneous brain activity in chronic tinnitus patients revealed by resting-state functional MRI. Neuroimage Clin. 6, 222-228. doi: 10.1016/j.nicl.2014.09.011

Damasio, A. R., Everitt, B., and Bishop, D. (1996). The somatic marker hypothesis and the possible functions of the prefrontal cortex [and discussion]. Philos. Trans. R. Soc. B Biol. Sci. 351, 1413-1420. doi: 10.1098/rstb.1996.0125

Davies, J., Gander, P., Andrews, M., and Hall, D. (2014). Auditory network connectivity in tinnitus patients: a resting-state fMRI study. Int. J. Audiol. 53, 192-198. doi: 10.3109/14992027.2013.846482

De Ridder, D., Elgoyhen, A. B., Romo, R., and Langguth, B. (2011). Phantom percepts: tinnitus and pain as persisting aversive memory networks. Proc. Natl. Acad. Sci. U S A 108, 8075-8080. doi: 10.1073/pnas.1018466108

De Ridder, D., Vanneste, S., Weisz, N., Londero, A., Schlee, W., Elgoyhen, A. B., et al. (2014). An integrative model of auditory phantom perception: tinnitus as a unified percept of interacting separable subnetworks. Neurosci. Biobehav. Rev. 44, 16-32. doi: 10.1016/j.neubiorev.2013.03.021

Di Martino, A., Zuo, X.-N., Kelly, C., Grzadzinski, R., Mennes, M., Schvarcz, A., et al. (2013). Shared and distinct intrinsic functional network centrality in autism and attention-deficit/hyperactivity disorder. Biol. Psychiatry 74, 623-632. doi: 10.1016/j.biopsych.2013.02.011

Fox, M. D., and Raichle, M. E. (2007). Spontaneous fluctuations in brain activity observed with functional magnetic resonance imaging. Nat. Rev. Neurosci. 8, 700-711. doi: 10.1038/nrn2201
Geven, L. I., de Kleine, E., Willemsen, A. T., and van Dijk, P. (2014). Asymmetry in primary auditory cortex activity in tinnitus patients and controls. Neuroscience 256, 117-125. doi: 10.1016/j.neuroscience.2013.10.015

Giraud, A. L., Chéry-Croze, S., Fischer, G., Fischer, C., Vighetto, A., Grégoire, M.-C., et al. (1999). A selective imaging of tinnitus. Neuroreport 10, 1-5. doi: 10. 1097/00001756-199901180-00001

Granger, C. W. (1969). Investigating causal relations by econometric models and cross-spectral methods. Econometrica 37, 424-438. doi: 10.2307/ 1912791

Gu, J. W., Halpin, C. F., Nam, E.-C., Levine, R. A., and Melcher, J. R. (2010). Tinnitus, diminished sound-level tolerance and elevated auditory activity in humans with clinically normal hearing sensitivity. J. Neurophysiol. 104, 3361-3370. doi: 10.1152/jn.00226.2010

Guo, W., Liu, F., Liu, J., Yu, L., Zhang, J., Zhang, Z., et al. (2015a). Abnormal causal connectivity by structural deficits in first-episode, drug-naive schizophrenia at rest. Schizophr. Bull. 41, 57-65. doi: 10.1093/schbul/sbu126

Guo, W., Liu, F., Zhang, Z., Liu, J., Yu, M., Zhang, J., et al. (2015b). Unidirectionally affected causal connectivity of cortico-limbic-cerebellar circuit by structural deficits in drug-naive major depressive disorder. J. Affect. Disord. 172, 410-416. doi: 10.1016/j.jad.2014.10.019

Haller, S., Birbaumer, N., and Veit, R. (2010). Real-time fMRI feedback training may improve chronic tinnitus. Eur. Radiol. 20, 696-703. doi: 10.1007/s00330009-1595-z

Henry, J. A., Roberts, L. E., Caspary, D. M., Theodoroff, S. M., and Salvi, R. J. (2014). Underlying mechanisms of tinnitus: review and clinical implications. J. Am. Acad. Audiol. 25, 5-22. doi: 10.3766/jaaa.25.1.2

Hinkley, L. B., Mizuiri, D., Hong, O., Nagarajan, S. S., and Cheung, S. W. (2015). Increased striatal functional connectivity with auditory cortex in tinnitus. Front. Hum. Neurosci. 9:568. doi: 10.3389/fnhum.2015.00568

Jackler, R. K., and Whinney, D. (2001). A century of eighth nerve surgery. Otol. Neurotol. 22, 401-416. doi: 10.1097/00129492-200109000-00029

Jastreboff, P. J. (1990). Phantom auditory perception (tinnitus): mechanisms of generation and perception. Neurosci. Res. 8, 221-254. doi: 10.1016/01680102(90)90031-9

Jastreboff, P. J., and Hazell, J. W. (2008). Tinnitus Retraining Therapy: Implementing the Neurophysiological Model. Cambridge, UK: Cambridge University Press.

Kaltenbach, J. A., Zhang, J., and Finlayson, P. (2005). Tinnitus as a plastic phenomenon and its possible neural underpinnings in the dorsal cochlear nucleus. Hear. Res. 206, 200-226. doi: 10.1016/j.heares.2005.02.013

Khalfa, S., Dubal, S., Veuillet, E., Perez-Diaz, F., Jouvent, R., and Collet, L. (2002). Psychometric normalization of a hyperacusis questionnaire. ORL J. Otorhinolaryngol. Relat. Spec. 64, 436-442. doi: 10.1159/000067570

Kim, J.-Y., Kim, Y.-H., Lee, S., Seo, J.-H., Song, H.-J., Cho, J. H., et al. (2012). Alteration of functional connectivity in tinnitus brain revealed by resting-state fMRI? a pilot study. Int. J. Audiol. 51, 413-417. doi: 10.3109/14992027.2011. 652677

Kringelbach, M. L. (2005). The human orbitofrontal cortex: linking reward to hedonic experience. Nat. Rev. Neurosci. 6, 691-702. doi: 10.1038/nrn1747

Kuk, F. K., Tyler, R. S., Russell, D., and Jordan, H. (1990). The psychometric properties of a tinnitus handicap questionnaire. Ear Hear. 11, 434-445. doi: 10. 1097/00003446-199012000-00005

Langguth, B., Eichhammer, P., Kreutzer, A., Maenner, P., Marienhagen, J., Kleinjung, T., et al. (2006). The impact of auditory cortex activity on characterizing and treating patients with chronic tinnitus-first results from a PET study. Acta Otolaryngol. Suppl. 126, 84-88. doi: 10. 1080/03655230600895317

Leaver, A. M., Renier, L., Chevillet, M. A., Morgan, S., Kim, H. J., and Rauschecker, J. P. (2011). Dysregulation of limbic and auditory networks in tinnitus. Neuron 69, 33-43. doi: 10.1016/j.neuron.2010.12.002

Leaver, A. M., Turesky, T. K., Seydell-Greenwald, A., Morgan, S., Kim, H. J., and Rauschecker, J. P. (2016). Intrinsic network activity in tinnitus investigated using functional MRI. Hum. Brain Mapp. doi: 10.1002/hbm.23204 [Epub ahead of print].

Lenarz, T., Schreiner, C., Snyder, R. L., and Ernst, A. (1993). Neural mechanisms of tinnitus. Eur. Arch. Otorhinolaryngol. 249, 441-446. doi: 10.1007/ bf00168851

Leske, M. C. (1981). Prevalence estimates of communicative disorders in the US Language, hearing and vestibular disorders. ASHA 23, 229-237. 
Lewis, J. W., Beauchamp, M. S., and DeYoe, E. A. (2000). A comparison of visual and auditory motion processing in human cerebral cortex. Cereb. Cortex 10, 873-888. doi: 10.1093/cercor/10.9.873

Lockwood, A. H., Salvi, R. J., and Burkard, R. F. (2002). Tinnitus. N. Engl. J. Med. 347, 904-910. doi: 10.1056/NEJMra013395

Lockwood, A. H., Salvi, R., Coad, M., Towsley, M., Wack, D., and Murphy, B. (1998). The functional neuroanatomy of tinnitus Evidence for limbic system links and neural plasticity. Neurology 50, 114-120. doi: 10.1212/ wnl.50.1.114

Logothetis, N. K., Murayama, Y., Augath, M., Steffen, T., Werner, J., and Oeltermann, A. (2009). How not to study spontaneous activity. Neuroimage 45, 1080-1089. doi: 10.1016/j.neuroimage.2009.01.010

Logothetis, N. K., Pauls, J., Augath, M., Trinath, T., and Oeltermann, A. (2001). Neurophysiological investigation of the basis of the fMRI signal. Nature 412, 150-157. doi: 10.1038/35084005

Mathiak, K., Menning, H., Hertrich, I., Mathiak, K. A., Zvyagintsev, M., and Ackermann, H. (2007). Who is telling what from where? A functional magnetic resonance imaging study. Neuroreport 18, 405-409. doi: 10.1097/wnr. 0b013e328013cec 4

Maudoux, A., Lefebvre, P., Cabay, J.-E., Demertzi, A., Vanhaudenhuyse, A., Laureys, S., et al. (2012a). Auditory resting-state network connectivity in tinnitus: a functional MRI study. PLoS One 7:e36222. doi: 10.1371/journal. pone. 0036222

Maudoux, A., Lefebvre, P., Cabay, J.-E., Demertzi, A., Vanhaudenhuyse, A., Laureys, S., et al. (2012b). Connectivity graph analysis of the auditory resting state network in tinnitus. Brain Res. 1485, 10-21. doi: 10.1016/j.brainres.2012. 05.006

McFadden, D. (1982). Tinnitus: Facts, Theories and Treatments. Washington, DC: National Academies.

Meikle, M. B. (1997). Electronic access to tinnitus data: the oregon tinnitus data archive. Otolaryngol. Head Neck Surg. 117, 698-700. doi: 10.1016/s01945998(97)70055-x

Melloni, L., Molina, C., Pena, M., Torres, D., Singer, W., and Rodriguez, E. (2007). Synchronization of neural activity across cortical areas correlates with conscious perception. J. Neurosci. 27, 2858-2865. doi: 10.1523/jneurosci.462306.2007

Minami, S. B., Oishi, N., Watabe, T., Uno, K., Kaga, K., and Ogawa, K. (2015). Auditory resting-state functional connectivity in tinnitus and modulation with transcranial direct current stimulation. Acta Otolaryngol. 135, 1286-1292. doi: 10.3109/00016489.2015.1068952

Mirz, F., Pedersen, B., Ishizu, K., Johannsen, P., Ovesen, T., Stødkilde-Jørgensen, H., et al. (1999). Positron emission tomography of cortical centers of tinnitus. Hear. Res. 134, 133-144. doi: 10.1016/s0378-5955(99)00075-1

Mühlau, M., Rauschecker, J., Oestreicher, E., Gaser, C., Röttinger, M., Wohlschläger, A., et al. (2006). Structural brain changes in tinnitus. Cereb. Cortex 16, 1283-1288. doi: 10.1093/cercor/bhj070

Murphy, K., Birn, R. M., Handwerker, D. A., Jones, T. B., and Bandettini, P. A. (2009). The impact of global signal regression on resting state correlations: are anti-correlated networks introduced? Neuroimage 44, 893-905. doi: 10.1016/j. neuroimage.2008.09.036

Murray, M. M., Molholm, S., Michel, C. M., Heslenfeld, D. J., Ritter, W., Javitt, D. C., et al. (2005). Grabbing your ear: rapid auditory-somatosensory multisensory interactions in low-level sensory cortices are not constrained by stimulus alignment. Cereb. Cortex 15, 963-974. doi: 10.1093/cercor/ bhh197

Osaki, Y., Nishimura, H., Takasawa, M., Imaizumi, M., Kawashima, T., Iwaki, T., et al. (2005). Neural mechanism of residual inhibition of tinnitus in cochlear implant users. Neuroreport 16, 1625-1628. doi: 10.1097/01.wnr.0000183899. 85277.08

Petacchi, A., Laird, A. R., Fox, P. T., and Bower, J. M. (2005). Cerebellum and auditory function: an ALE meta-analysis of functional neuroimaging studies. Hum. Brain Mapp. 25, 118-128. doi: 10.1002/hbm.20137

Qi, R., Zhang, L. J., Zhong, J., Zhang, Z., Ni, L., Jiao, Q., et al. (2013). Altered effective connectivity network of the basal ganglia in low-grade hepatic encephalopathy: a resting-state fMRI study with Granger causality analysis. PLoS One 8:e53677. doi: 10.1371/journal.pone.0053677

Rauschecker, J. P., Leaver, A. M., and Mühlau, M. (2010). Tuning out the noise: limbic-auditory interactions in tinnitus. Neuron 66, 819-826. doi: 10.1016/j. neuron.2010.04.032
Roberts, L. E., Eggermont, J. J., Caspary, D. M., Shore, S. E., Melcher, J. R., and Kaltenbach, J. A. (2010). Ringing ears: the neuroscience of tinnitus. J. Neurosci. 30, 14972-14979. doi: 10.1523/JNEUROSCI.4028-10.2010

Roland, P. E., Larsen, B., Lassen, N., and Skinhøj, E. (1980). Supplementary motor area and other cortical areas in organization of voluntary movements in man. J. Neurophysiol. 43, 118-136.

Rolls, E. T. (2004). The functions of the orbitofrontal cortex. Brain Cogn. 55, 11-29. doi: 10.1016/S0278-2626(03)00277-X

Schecklmann, M., Landgrebe, M., Poeppl, T. B., Kreuzer, P., Männer, P., Marienhagen, J., et al. (2013). Neural correlates of tinnitus duration and distress: a positron emission tomography study. Hum. Brain Mapp. 34, 233-240. doi: 10.1002/hbm.21426

Schlee, W., Mueller, N., Hartmann, T., Keil, J., Lorenz, I., and Weisz, N. (2009). Mapping cortical hubs in tinnitus. BMC Biol. 7:80. doi: 10.1186/1741-7007-780

Schmidt, S. A., Akrofi, K., Carpenter-Thompson, J. R., and Husain, F. T. (2013). Default mode, dorsal attention and auditory resting state networks exhibit differential functional connectivity in tinnitus and hearing loss. PLoS One 8:e76488. doi: 10.1371/journal.pone.0076488

Shulman, A., and Strashun, A. (1998). Descending auditory system/cerebellum/tinnitus. Int. Tinnitus J. 5, 92-106.

Smith, S. M., Miller, K. L., Salimi-Khorshidi, G., Webster, M., Beckmann, C. F., Nichols, T. E., et al. (2011). Network modelling methods for FMRI. Neuroimage 54, 875-891. doi: 10.1016/j.neuroimage.2010.08.063

Smits, M., Kovacs, S., De Ridder, D., Peeters, R. R., Van Hecke, P., and Sunaert, S. (2007). Lateralization of functional magnetic resonance imaging (fMRI) activation in the auditory pathway of patients with lateralized tinnitus. Neuroradiology 49, 669-679. doi: 10.1007/s00234-007-0231-3

Song, J.-J., De Ridder, D., Weisz, N., Schlee, W., Van de Heyning, P., and Vanneste, S. (2014). Hyperacusis-associated pathological resting-state brain oscillations in the tinnitus brain: a hyperresponsiveness network with paradoxically inactive auditory cortex. Brain Struct. Funct. 219, 1113-1128. doi: 10.1007/s00429-0130555-1

Sporns, O., Honey, C. J., and Kötter, R. (2007). Identification and classification of hubs in brain networks. PLoS One 2:e1049. doi: 10.1371/journal.pone. 0001049

Ueyama, T., Donishi, T., Ukai, S., Ikeda, Y., Hotomi, M., Yamanaka, N., et al. (2013). Brain regions responsible for tinnitus distress and loudness: a restingstate fMRI study. PLoS One 8:e67778. doi: 10.1371/journal.pone.0067778

Vanneste, S., and De Ridder, D. (2012). The auditory and non-auditory brain areas involved in tinnitus. An emergent property of multiple parallel overlapping subnetworks. Front. Syst. Neurosci. 6:31. doi: 10.3389/fnsys.2012.00031

Vanneste, S., Joos, K., and De Ridder, D. (2012). Prefrontal cortex based sex differences in tinnitus perception: same tinnitus intensity, same tinnitus distress, different mood. PLoS One 7:e31182. doi: 10.1371/journal.pone. 0031182

Vanneste, S., Plazier, M., Van der Loo, E., Van de Heyning, P., Congedo, M., and De Ridder, D. (2010). The neural correlates of tinnitus-related distress Neuroimage 52, 470-480. doi: 10.1016/j.neuroimage.2010.04.029

Vanneste, S., Plazier, M., van der Loo, E., Van de Heyning, P., and De Ridder, D. (2011). The difference between uni-and bilateral auditory phantom percept. Clin. Neurophysiol. 122, 578-587. doi: 10.1016/j.clinph.2010.07.022

Vincent, J. L., Kahn, I., Snyder, A. Z., Raichle, M. E., and Buckner, R. L. (2008). Evidence for a frontoparietal control system revealed by intrinsic functional connectivity. J. Neurophysiol. 100, 3328-3342. doi: 10.1152/jn. 90355.2008

Voisin, J., Bidet-Caulet, A., Bertrand, O., and Fonlupt, P. (2006). Listening in silence activates auditory areas: a functional magnetic resonance imaging study. J. Neurosci. 26, 273-278. doi: 10.1523/jneurosci.2967-05.2006

Webb, J. T., Ferguson, M. A., Nielsen, J. A., and Anderson, J. S. (2013). BOLD granger causality reflects vascular anatomy. PLoS One 8:e84279. doi: 10 1371/journal.pone.0084279

Wineland, A. M., Burton, H., and Piccirillo, J. (2012). Functional connectivity networks in nonbothersome tinnitus. Otolaryngol. Head Neck Surg. 147, 900-906. doi: 10.1177/0194599812451414

Wunderlich, A. P., Schönfeldt-Lecuona, C., Wolf, R. C., Dorn, K., Bachor, E., and Freund, W. (2010). Cortical activation during a pitch discrimination task in tinnitus patients and controls-an fMRI study. Audiol. Neurootol. 15, 137-148. doi: $10.1159 / 000241094$ 
Yan, C.-G., Craddock, R. C., Zuo, X.-N., Zang, Y.-F., and Milham, M. P. (2013). Standardizing the intrinsic brain: towards robust measurement of interindividual variation in 1000 functional connectomes. Neuroimage 80, 246-262. doi: 10.1016/j.neuroimage.2013.04.081

Zhang, J., Chen, Y.-C., Feng, X., Yang, M., Liu, B., Qian, C., et al. (2015). Impairments of thalamic resting-state functional connectivity in patients with chronic tinnitus. Eur. J. Radiol. 84, 1277-1284. doi: 10.1016/j.ejrad. 2015.04.006

Zang, Z.-X., Yan, C.-G., Dong, Z.-Y., Huang, J., and Zang, Y.-F. (2012). Granger causality analysis implementation on MATLAB: a graphic user interface toolkit for fMRI data processing. J. Neurosci. Methods 203, 418-426. doi: 10.1016/j. jneumeth.2011.10.006

Zhong, Y., Huang, L., Cai, S., Zhang, Y., von Deneen, K. M., Ren, A., et al. (2014). Altered effective connectivity patterns of the default mode network in Alzheimer's disease: an fMRI study. Neurosci. Lett. 578, 171-175. doi: 10.1016/j. neulet.2014.06.043

Zhou, Z., Wang, X., Klahr, N. J., Liu, W., Arias, D., Liu, H., et al. (2011). A conditional Granger causality model approach for group analysis in functional magnetic resonance imaging. Magn. Reson. Imaging 29, 418-433. doi: 10. 1016/j.mri.2010.10.008
Zung, W. W. (1971). A rating instrument for anxiety disorders. Psychosomatics 12, 371-379. doi: 10.1016/s0033-3182(71)71479-0

Zung, W. (1986). "Zung self-rating depression scale and depression status inventory," in Assessment of Depression, eds N. Sartorius and T. A. Ban (Berlin: Springer), 221-231.

Zuo, X.-N., Ehmke, R., Mennes, M., Imperati, D., Castellanos, F. X., Sporns, O., et al. (2012). Network centrality in the human functional connectome. Cereb. Cortex 22, 1862-1875. doi: 10.1093/cercor/bhr269

Conflict of Interest Statement: The authors declare that the research was conducted in the absence of any commercial or financial relationships that could be construed as a potential conflict of interest.

Copyright (c) 2016 Chen, Feng, Xu, Mao, Xia, Ren and Yin. This is an open-access article distributed under the terms of the Creative Commons Attribution License (CC BY). The use, distribution and reproduction in other forums is permitted, provided the original author(s) or licensor are credited and that the original publication in this journal is cited, in accordance with accepted academic practice. No use, distribution or reproduction is permitted which does not comply with these terms. 